University of Nebraska - Lincoln

DigitalCommons@University of Nebraska - Lincoln

April 1997

\title{
Interlayer coupling in NiFe/Mo multilayers and effect of Fe underlayer
}

\author{
M.L. Yan \\ University of Nebraska - Lincoln \\ Z.S. Shan \\ University of Nebraska - Lincoln \\ David J. Sellmyer \\ University of Nebraska-Lincoln, dsellmyer@unl.edu \\ W.Y. Lai \\ Institute of Physics, Chinese Academy of Sciences, Beijing 100080, People's Republic of China
}

Follow this and additional works at: https://digitalcommons.unl.edu/physicssellmyer

Part of the Physics Commons

Yan, M.L.; Shan, Z.S.; Sellmyer, David J.; and Lai, W.Y., "Interlayer coupling in NiFe/Mo multilayers and effect of Fe underlayer" (1997). David Sellmyer Publications. 84.

https://digitalcommons.unl.edu/physicssellmyer/84

This Article is brought to you for free and open access by the Research Papers in Physics and Astronomy at DigitalCommons@University of Nebraska - Lincoln. It has been accepted for inclusion in David Sellmyer Publications by an authorized administrator of DigitalCommons@University of Nebraska - Lincoln. 


\title{
Interlayer coupling in NiFe/Mo multilayers and effect of Fe underlayer
}

\author{
M. L. Yan, ${ }^{\text {a) }}$ Z. S. Shan, and D. J. Sellmyer \\ Center for Materials Research and Analysis, University of Nebraska, Lincoln, Nebraska 68588-0113 \\ W. Y. Lai \\ Institute of Physics, Chinese Academy of Sciences, Beijing 100080, People's Republic of China
}

\begin{abstract}
We report our studies of exchange coupling in NiFe/Mo multilayers with and without Fe underlayer. The interlayer coupling of NiFe layers across the Mo spacer as well as the magnetic exchange coupling between Fe underlayer and the NiFe/Mo multilayers were observed: The interlayer coupling in $\mathrm{NiFe} / \mathrm{Mo}$ multilayers exhibits the typical oscillations as a function of the Mo spacer with a period of about $11 \AA$, and the magnetic exchange coupling between Fe underlayer and the $\mathrm{NiFe} / \mathrm{Mo}$ multilayers is strongly dependent on the thickness of the Mo spacer $t_{\mathrm{Mo}}$ and the bilayer number $N$ of $\mathrm{NiFe} / \mathrm{Mo}$ multilayers. For the $\left[\mathrm{NiFe}(20 \AA) / \mathrm{Mo}\left(t_{\mathrm{Mo}}\right)\right]_{30}$ samples with a $100 \AA \mathrm{Fe}$ underlayer, the hysteresis loops with thinner $\operatorname{Mos} \operatorname{spacer}\left(t_{\mathrm{Mo}}<4 \AA\right.$ ) $)$ are single loops indicating that the Fe underlayer is strongly magnetic exchange coupled with the NiFe/Mo multilayers; whereas hysteresis loops with thicker Mo spacer $\left(t_{\mathrm{Mo}} \geqslant 5 \AA\right.$ ) show a two-magnetic-phase feature. For the $[\mathrm{NiFe}(20 \AA) / \mathrm{Mo}(10 \AA)]_{30}$ sample with $100 \AA \mathrm{Ae}$ underlayer, the hysteresis loops are single loops for bilayer number $N<15$, while hysteresis loops show the two-phase feature for $N \geqslant 15$. (C) 1997 American Institute of Physics. [S0021-8979(97)48908-9]
\end{abstract}

Recently, extensive theoretical and experimental studies have been devoted to multilayers composed of alternating magnetic and nonmagnetic layers. In part, this interest has been generated by the antiferromagnetic (AFM) interlayer coupling and giant magnetoresistance (GMR). The wellknown systems are $\mathrm{Fe} / \mathrm{Cr}$ and $\mathrm{Co} / \mathrm{Cu} .{ }^{1-4}$ Oscillation in interlayer coupling as a function of the nonmagnetic spacer thickness has been demonstrated to be a general property for most magnetic/nonmagnetic transition metal systems. ${ }^{5}$ The short period oscillations have been found besides the long period oscillations that have been identified in multilayers and sandwiches. In our previous articles ${ }^{6,7}$ the typical oscillations of the interlayer coupling as a function of the Mo spacer thickness were observed in the $\mathrm{Fe} / \mathrm{Mo}$ and $\mathrm{NiFe} / \mathrm{Mo}$ multilayers. The larger phase shift in the oscillatory interlayer coupling across Mo spacer was also reported. In this article we describe the experimental results of coupling on NiFe/Mo multilayers with and without $\mathrm{Fe}$ underlayer. Both the interlayer coupling between NiFe magnetic layers across the Mo spacer and the magnetic exchange coupling between $\mathrm{Fe}$ underlayer and the $\mathrm{NiFe} / \mathrm{Mo}$ multilayers were observed.

$\left[\mathrm{NiFe}(20 \AA) / \mathrm{Mo}\left(t_{\mathrm{Mo}}\right)\right]_{N}$ multilayers with and without $\mathrm{Fe}(100 \AA)$ underlayer were prepared in a magnetron sputtering system with the base pressure better than $3 \times 10^{-5} \mathrm{~Pa}$. The composition of the NiFe target was $\mathrm{Ni} 80 \% \mathrm{Fe} 20 \%$, and material of the Mo target was $99.9 \%$ nominal purity. The periodicity of chemical composition modulation and crystallographic structure of multilayers were confirmed by x-ray diffraction (XRD). Low-angle $\mathrm{x}$-ray diffraction revealed that superlattice peaks appear in the reflectivity spectra for all multilayered samples, and the corresponding high-angle $\mathrm{x}$-ray-diffraction spectra for those samples show that the structure of the $\mathrm{NiFe}$ is fcc(111) texture, whereas that of the

\footnotetext{
${ }^{a)}$ On leave from: State Key Laboratory for Magnetism, Institute of Physics, Chinese Academy of Sciences, Beijing 100080, People's Republic of China.
}

Mo layer is bcc(110) texture. The coupling was investigated at room temperature by measuring the magnetic hysteresis loops using a vibrating sample magnetometer (VSM) and AGFM with the applied field in the film plan.

Figure 1 shows variation of the saturation field $H_{s}$ as a function of the Mo spacer thickness $t_{\mathrm{Mo}}$ for $[\mathrm{NiFe}(20$ $\left.\AA) / \mathrm{Mo}\left(t_{\mathrm{Mo}}\right)\right]_{30}$ multilayers with and without Fe underlayer. As shown in Fig. 1, $H_{s}$ oscillates with the variation of the Mo spacer thickness $t_{\mathrm{Mo}}$. The period of oscillation is about $11 \AA$ A. This oscillation is indicative of an oscillation in interlayer coupling between the adjacent NiFe layers from AFM to FM state. The maximum of those oscillations corresponds to the AFM state, and minimum corresponds to the FM one. As the thickness of the Mo spacer increases, the saturation field $H_{s}$ decreases indicating that the AFM coupling strength falls off rapidly. This behavior is similar to that reported in most multilayered system. ${ }^{3,8}$ In comparison with $H_{s}$ in Fig. 1 , we can see that in the AFM coupling state saturation field $H_{s}$ is nearly the same for both series of NiFe/Mo multilayer except the third AFM state, whereas the obvious difference between saturation field $H_{s}$ is seen at the FM state, especially

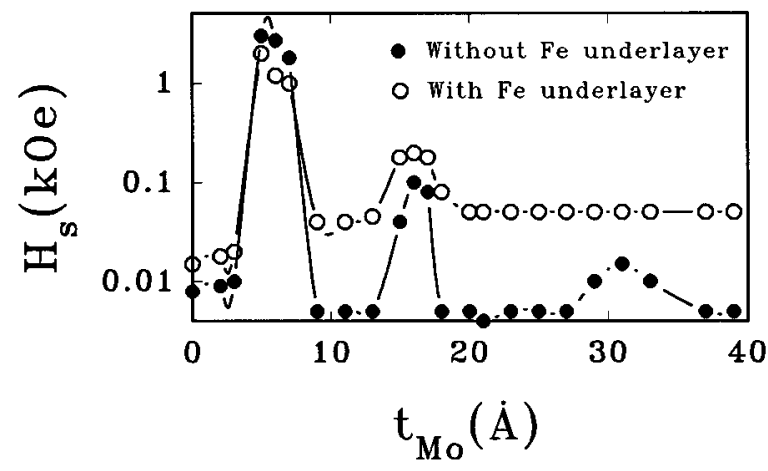

FIG. 1. Variation of saturation field $H_{s}$ as a function of the Mo spacer thickness for $\mathrm{NiFe} / \mathrm{Mo}$ multilayers with and without Fe underlayer. Open and solid symbols are samples with and without Fe underlayer, respectively. 


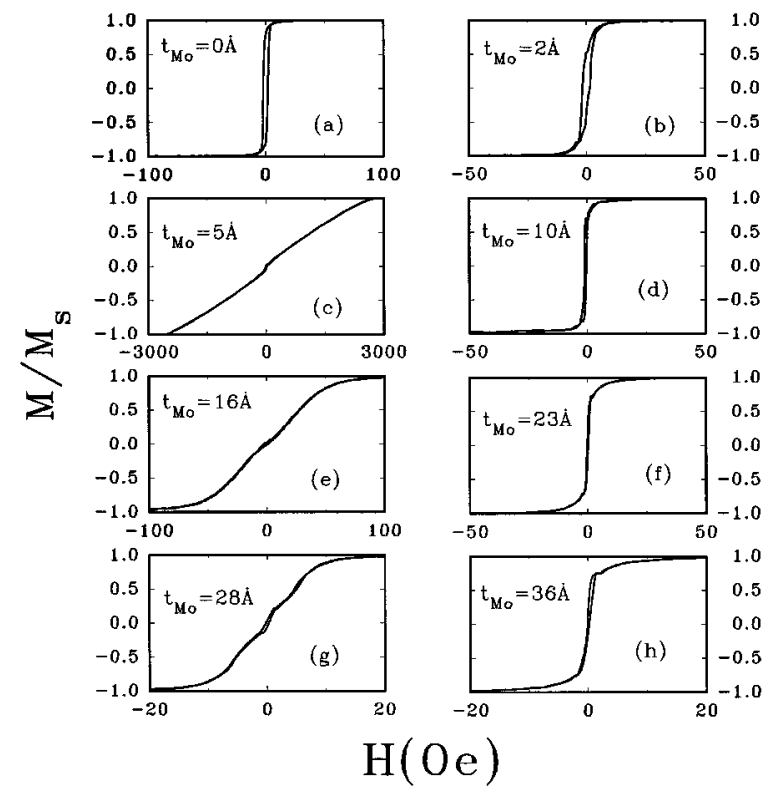

FIG. 2. The room-temperature hysteresis loops are shown for $[\mathrm{NiFe}(20$ $\left.\AA) / \mathrm{Mo}\left(t_{\mathrm{Mo}}\right)\right]_{30}$ multilayers without Fe underlayer.

for thicker Mo spacer. The $H_{s}$ in multilayers with Fe underlayer is higher than that of the multilayers without Fe underlayer.

A compilation of measured hysteresis loops of $[\mathrm{NiFe}(20$ $\left.\AA) / \mathrm{Mo}\left(t_{\mathrm{Mo}}\right)\right]_{30}$ multilayers without underlayer, which was obtained at the indicated thickness of the Mo spacer, is shown in Fig. 2. It is seen that the hysteresis loop [Fig. 2(a)] of $t_{\mathrm{Mo}}=0$, i.e., a pure $600 \AA \mathrm{NiFe}$ film, which exhibits a larger remenence $M_{r}$ and very small saturation field $H_{s}$, is ferromagnetic. Similarly, the hysteresis loops of 2(b), 2(d), 2(f), and 2(h) for $t_{\mathrm{Mo}}=2,10,23$, and $36 \AA$ have larger remenence $M_{r}$ and small $H_{s}$, indicating that neighboring magnetic layers of those samples are ferromagnetically coupled. However, loops 2(c), 2(e), and 2(g) with $t_{\mathrm{Mo}}=5,16$, and 28 $\AA$ show different features: The remenence $M_{r}$ of the loops 2(c), 2(d), and 2(g) is nearly reduced to zero and the saturation field $H_{s}$ is large. Notice the different scale on the abscissa. The magnetization behaviors of those samples are typical AFM interlayer coupling. Figure 3 shows the compilation of measured hysteresis loops of $[\mathrm{NiFe}(20$ $\left.\AA) / \mathrm{Mo}\left(t_{\mathrm{Mo}}\right)\right]_{30}$ multilayers as shown in Fig. 2, but with a 100 A Fe underlayer. By comparing Fig. 3 with Fig. 2, we can see that in the AFM state the remenence/saturation magnetization ratio $M_{r} / M_{s}$ of NiFe multilayers with an Fe underlayer is relatively high in addition to a high saturation field $H_{s}$; in the FM state loops show a step jump of two transitions [Figs. 2(d), 2(f), and 2(h)]. These magnetic behaviors are very different from that of antiferromagnetically or ferromagnetically coupled $\mathrm{NiFe} / \mathrm{Mo}$ multilayers without Fe underlayer: For antiferromagnetically coupled NiFe/Mo multilayers as shown in Fig. 2, the ratios $M_{r} / M_{s}$ are rather small due to the moment of magnetic layers is aligned antiparallel in the zero field; and for ferromagnetically coupled multilayers, high $M_{r} / M_{s}$ is due to the moment of the magnetic layers is still aligned parallel in the zero field. Clearly, Fe underlayer affects the detailed shape of the hysteresis loops. The

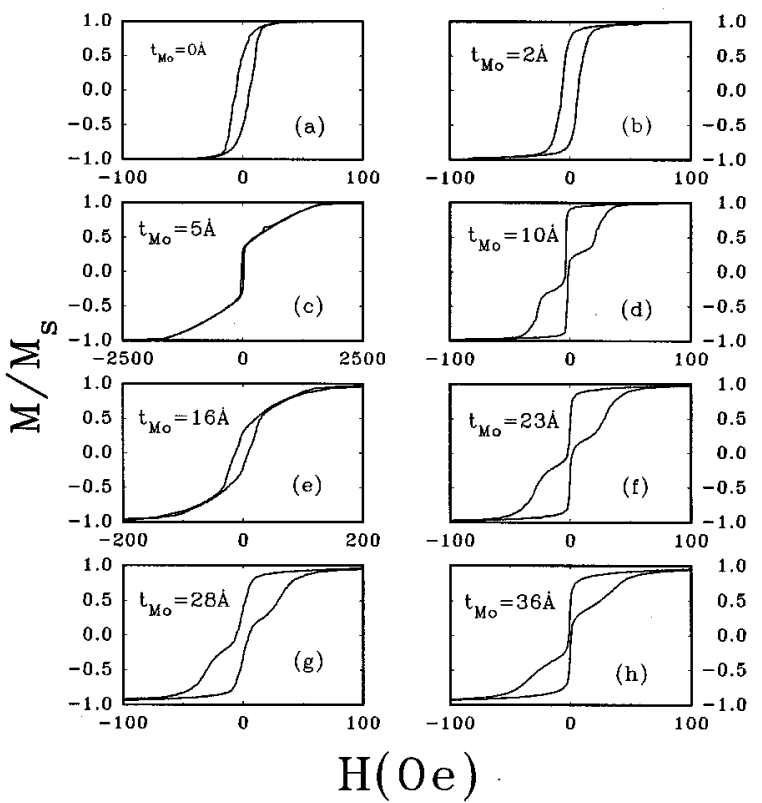

FIG. 3. The room-temperature hysteresis loops of $\left[\mathrm{NiFe}(20 \AA) / \mathrm{Mo}\left(t_{\mathrm{Mo}}\right)\right]_{30}$ multilayers with a $100 \AA$ Fe underlayer.

magnetic measurement showed that coercive force of a 100 $\AA$ Fe film was larger and loop was "wider" compared with that of $\mathrm{NiFe} / \mathrm{Mo}$ multilayers. Here we show that consideration of magnetic coupling assists the interpretation of complex hysteresis loops.

(1) For samples with thinner Mo spacers $\left(t_{\mathrm{Mo}}=1,2,3\right.$, and $4 \AA$ ) the adjacent NiFe layers are coupled across Mo spacer ferromagnetically, and the interlayer coupling strength is vary strong. At a high saturation field, all $\mathrm{NiFe}$ layers in multilayers as well as Fe underlayer align their moments in the field direction. When the field is decreased to zero, and then increased in opposite direction, the moments of both the NiFe/Mo multilayer with low coercive force and the Fe underlayer with high coercive force are gradually reversed together, due to the $\mathrm{Fe}$ underlayer being exchange coupled with the NiFe/Mo multilayer. As a consequence, loops are simple single loops [Fig. (3b)].

(2) For the samples with thicker Mo spacer $\left(t_{\mathrm{Mo}} \geqslant 5 \AA\right)$ the interlayer coupling strength of adjacent $\mathrm{NiFe}$ layers across Mo spacer becomes relatively weaker in comparison with thinner Mo spacer. In the case of NiFe layers coupled ferromagnetically [Figs. 3(d), 3(f), and 3(h)], the moment of the Fe underlayer is oriented parallel to that of NiFe layers at zero field and does not reverse unless an large enough magnetic field is applied in the opposite direction. When a small magnetic field is applied in the opposite direction, because of relatively weaker interlayer coupling between NiFe layers, the moment of part of the NiFe layers that is far away from Fe underlayer is reversed at first, due to the low coercive force. As a result, magnetization shows a rapid decrease, and a knee appears on the loops. By further increasing the magnetic field, the moment of $\mathrm{NiFe}$ layers that is near the $\mathrm{Fe}$ underlayer and the moment of the $\mathrm{Fe}$ underlayer begin to reverse as well, due to the exchange coupling between them. As a result, magnetization of the loops appears a step jump, showing a two-phase feature. 


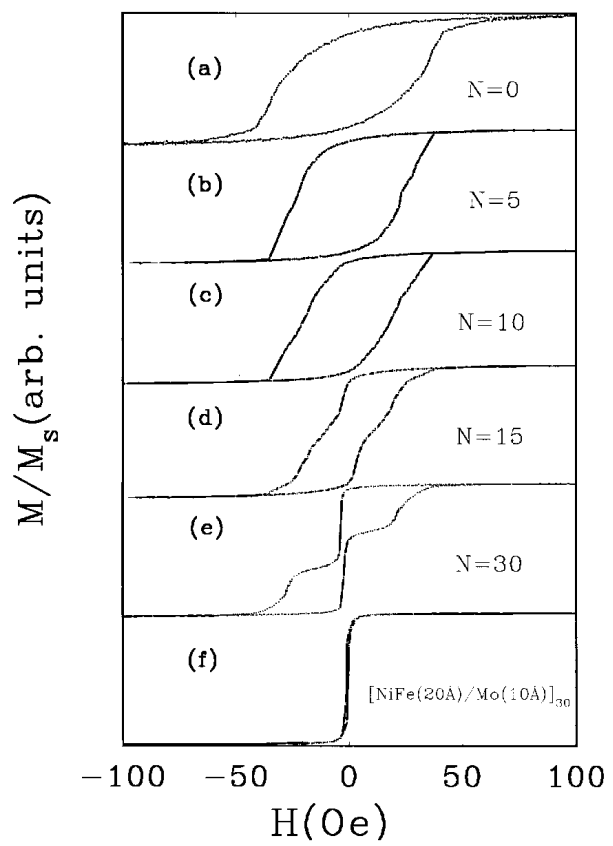

FIG. 4. The hysteresis loops of $[\mathrm{NiFe}(20 \AA) / \mathrm{Mo}(10 \AA)]_{N}$ multilayers with a $100 \AA$ Fe underlayer for varying $N$.

(3) In the case of NiFe layers coupled antiferromagnetically, when magnetic field decreases from the saturated state, the moments of every other $\mathrm{NiFe}$ layer are gradually reversed. At the zero field, the $M_{r}$ should be very small because AFM coupling aligns the moment of the NiFe layers antiparallel. It is evident that the higher $M_{r}$ values [Figs. 2(c), 2(e)] comes from the contribution of the $\mathrm{Fe}(100 \AA)$ underlayer. For thicker Mo spacer, the interlayer coupling strength reduces more. The loop shows a steplike behavior [Fig. 2(g)], although adjacent NiFe layers are coupled antiferromagnetically.

The magnetic exchange coupling is also observed to be dependent on the bilayer numbers $N$ of the NiFe/Mo multilayers. Figure 4 shows the hysteresis loops of $[\mathrm{NiFe}(20 \AA) /$ $\mathrm{Mo}(10 \AA)]_{N}$ multilayers with a $100 \AA \mathrm{Fe}$ underlayer for varying $N$. The loop of $N=0$, i.e., a pure $100 \AA$ Fe film [Fig. $4(\mathrm{a})]$ and the loop of $[\mathrm{NiFe}(20 \AA) / \mathrm{Mo}(10 \AA)]_{30}$ multilayer without $\mathrm{Fe}$ underlayer are also shown for comparing. The coercive force of $\mathrm{Fe}$ film is higher than that of $\mathrm{NiFe} / \mathrm{Mo}$ multilayer. The coercive forces for $100 \AA \mathrm{Fe}$ films and the $[\mathrm{NiFe}(20 \AA) / \mathrm{Mo}(10 \AA)]_{30}$ multilayer are 50 and $0.1 \mathrm{Oe}$, respectively. The $M_{r} / M_{s}$ was 0.8 for $100 \AA \mathrm{Fe}$ film and 0.9 for $[\operatorname{NiFe}(20 \AA) / \mathrm{Mo}(10 \AA)]_{30}$ multilayer. Figures $4(\mathrm{~b})-4(\mathrm{e})$ show hysteresis loops for samples with $N=5-30$. As can be seen in this figure, the coercive force of samples decreases and $M_{r} / M_{s}$ increases when $N$ is increased, which reflects the variation of the magnetic exchange coupling. In the case of $N<15$ the loops are simple single loops, whereas typical two-phase loops are seen as $N \geqslant 15$. These results imply that the exchange coupling between the $\mathrm{Fe}$ underlayer and $\mathrm{NiFe} / \mathrm{Mo}$ multilayers is within this limited distance. The $\mathrm{Fe}$ underlayer is totally exchange coupled to $\mathrm{NiFe} / \mathrm{Mo}$ multilayer as $N<15$, whereas part of $N \geqslant 15$ in NiFe/Mo multilayers is not coupled with $\mathrm{Fe}$ underlayer. Thus, the part of $N \geqslant 15$ in NiFe/Mo multilayer is first reversed when an opposite field was applied from zero. As a result, the magnetization of the film falls off rapidly, and the hysteresis loop appears a two-phase behavior.

This work is supported by NSF in China and by NSF under Grant No. DMR-9623992 in the U.S. The authors are very grateful to Hongwei Jiang, Ailing Wang, and Wu Zheng for assistance in preparing samples.

${ }^{1}$ P. Grünberg, R. Schreiber, Y. Pang, M. B. Brodsky, and H. Sowers, Phys. Rev. Lett. 57, 2442 (1986).

${ }^{2}$ M. N. Baibich, J. M. Broto, A. Fert, F. Nguyen Van Dau, F. Petroff, P. Etienne, G. Creuzet, A. Friederich, and J. Chazelas, Phys. Rev. Lett. 61, 2472 (1988).

${ }^{3}$ D. H. Mosca, F. Petroff, A. Fert, P. A. Schroeder, W. P. Pratt, Jr., and R. Loloee, J. Magn. Magn. Mater. 94, L1 (1991).

${ }^{4}$ S. S. P. Parkin, R. Bhadra, and K. P. Roche, Phys. Rev. Lett. 66, 2152 (1991).

${ }^{5}$ S. S. P. Parkin, Phys. Rev. Lett. 67, 3598 (1991).

${ }^{6}$ M. L. Yan, W. Y. Lai, Y. Z. Wang, S. X. Li, and C. T. Yu, J. Appl. Phys. 77, 1816 (1995)

${ }^{7}$ M. L. Yan, W. Y. Lai, G. M. Luo, and Z. H. Mai, J. Phys., Condens. Matter 8, L711 (1996).

${ }^{8}$ S. S. P. Parkin, N. More, and K. P. Roche, Phys. Rev. Lett. 64, 2304 (1990) 